


\title{
EDUCACIÓN PARA LA DESCOLONIZACIÓN
}

\section{Education for decolonization}

\author{
César Augusto Mendoza Alba ${ }^{1}$, Daniel Fernando Rodríguez Rincón ${ }^{2}$ \\ ${ }^{1}$ IE San Pedro Claver, Chitaraque-Boyacá, 'IE Sochaquira abajo, Guayatá-Boyacá \\ Email: ${ }^{1}$ cesar.mendozaalba@uptc.edu.co, ${ }^{2}$ daniel.rodriguez17@uptc.edu.co
}

(Recibido el 28 de mayo de 2021 - Aceptado el 08 de junio de 2021)

\section{Resumen}

Cualquier sociedad que pretenda cambiar el rumbo de la historia, en contra del orden establecido, debe asumir la convicción de crear un nuevo esquema teórico para escribir una historia desde su praxis entendiendo la realidad histórico-social en particular; por ende, la descolonización del pensamiento forma parte de la superación del sentido común alineado a las dinámicas de enseñanza y aprendizaje entabladas en la educación y se presenta como alternativa para incidir conscientemente en la realidad, a partir de una acción social transformadora y prefigurada por los sujetos protagonistas del cambio de estructura generando un pensamiento crítico y pedagogías alternativas. En una sociedad que limita, cada vez más, las posibilidades de actuar por el bien común resultan de vital la convivencia solidaria para lograr la vida en paz y en una relación de equilibrio con los territorios donde se habita generando estrategias educativas desligadas a la imposición histórica de pensamiento en los claustros académicos y educativos. El reconocimiento de la diversidad histórico-cultural existente en el mundo y los distintos sentidos de la historia, expresados en cosmovisiones diferentes de modos de organizarse y vivir en sociedad, permite valorar la coexistencia de comunidades y pueblos que comparten hábitats y territorios y que se articulan entre ellos para cooperar mutuamente, complementarse e intercambiar recursos y capacidades, en una relación de reciprocidad orientada a la educación, el pensamiento descolonizado y armonía entre todas las personas dentro de contextos sociales predefinidos basados en estrategias educativas críticas.

Palabras clave: descolonización, pensamiento, historia, educación, cosmovisión.

\section{Abstract}

Any society that intends to change the course of history, against the established order, must assume the conviction of creating a new theoretical scheme to write a history from its praxis understanding the historical-social reality in particular; therefore, the decolonization of 
thought is part of the overcoming of common sense aligned to the dynamics of teaching and learning engaged in education and is presented as an alternative to consciously influence reality, from a transformative social action and prefigured by the protagonists of the change of structure generating a critical thinking and alternative pedagogies. In a society that limits, more and more, the possibilities of acting for the common good are vital coexistence in solidarity to achieve life in peace and in a relationship of balance with the territories where they live generating educational strategies unrelated to the historical imposition of thought in the academic and educational cloisters. The recognition of the historical-cultural diversity existing in the world and the different meanings of history, expressed in different worldviews of ways of organizing and living in society, allows us to value the coexistence of communities and people that share habitats and territories and that articulate among them to cooperate with each other, complement each other and exchange resources and capacities, in a relationship of reciprocity oriented, decolonized thinking and harmony among all people within predefined social contexts based on critical educational strategies.

Keywords: decolonization, thought, history, education, worldview.

\section{INTRODUCCIÓN}

Son múltiples las posturas que diversos autores han desarrollado a lo largo de los últimos 40 años, sobre el pensamiento crítico y las pedagogías críticas de América Latina, como un instrumento de transformación social y del resurgimiento de sujetos oprimidos, y a su vez han procurado develar el proceder y su impacto en las practicas docentes, no obstante, delimitar el alcance obtenido en América Latina, especialmente en Colombia se torna complejo, debido a la influencia de intereses dominadores y capitalistas, que segmentan los grupos y conllevan a la divergencia de pensamientos y en sus ideales de transformación, sumado a estos, se debe tener en cuenta las diversas aristas desde las cuales los procesos de transformación de los sujetos pueden llegar a desarrollarse y que ha librado sus propias batallas por tratar de posicionarse dentro del amplio espectro de la educación liberadora.

La formación de sujetos críticos debe surgir del conocimiento de sí mismo, del reconocer del contexto y principalmente de ser conscientes y críticos del proceder como individuos y las diversas afectaciones sociales, culturales políticas e incluso ambiental, es a partir de este reconocimiento que el individuo además de formarse, empieza la transformación de sus pares y así, contribuir en cierta medida en la transformación de la sociedad, que durante años se ha visto sometida a modelos dominadores. 
Es por esto que el propósito del presente escrito está encaminado hacia la comprensión de las diversas percepciones e ideologías y elementos que han contribuido a romper los modelos educativos de origen europeo y norte americano los cuales develan un insípido interés por la formación de sujetos libres, con la capacidad de reconocerse a sí mismo y a su entorno, reconocimiento que es fundamental para encaminar la educación a una verdadera transformación de su esencia. Así pues, el recorrido empezará por conocer el pensamiento de Foucault y a través de una linealidad temporal se irán estableciendo los elementos que den paso a la comprensión y a la construcción de un modelo propio de educación desde una visión globalizadora, que permita la elaboración de procesos de liberación y con el firme propósito de edificar de sujetos críticos e integrales.

\section{REFLEXIÓN CRÍTICA}

Toda empresa emancipadora surge de una actitud crítica y toda crítica es consecuencia de un proceso hermenéutico de construcción de significado. El ser humano se vale para su comprensión del mundo de las interpretaciones de la realidad realizadas por otras personas, interpretaciones que se legitiman en círculos académicos, en el fragor de procesos socio-científicos que bien pueden ser objeto de crítica en sí mismos.

La crítica en su acepción más intuitiva es una posición intelectual o racional en contra de un cuerpo teórico, es más una actitud de cuestionamiento que un cuerpo teórico opuesto a cierta ideología en cuestión y, en general, es una oposición al dogma y al poder emanado de constructos hegemónicos socialmente afianzados, en palabras de Foucault la crítica o asumir la "actitud crítica" se definiría como: una cierta manera de pensar, de decir, de actuar, una cierta relación con lo que existe, con lo que se sabe, con lo que se hace, una relación con la sociedad, con la cultura, una relación, también, con los otros (1995).

El trabajo de Foucault (1995), es bastante importante para construir una historia y una concepción histórica de la crítica en la humanidad, y en particular de la crítica al poder. Foucault establece un abordaje metodológico, denominado arqueológico por su naturaleza, en esta arqueología el investigador busca elementos comunes a varios contenidos producidos voluntaria o involuntariamente en el pasado para establecer categorías que den cuenta de las concepciones o conceptos más relevantes y menos explícitos en la antigüedad.

Con esta metodología, Focault (1995), desenmascara en primera medida el poder que podríamos denominar del soberano y que tiene su más clara representación en el ejercicio violento y jerárquico de la autoridad en el contexto de una monarquía o del feudalismo 
donde es evidente la localización real y simbólica del sujeto dominador y del dominado. Lo esencial en Focault (1995), es su demostración de la existencia del poder en otras formas como consecuencia de un proceso de evolución que demanda del poderoso una capacidad para ejercer el poder (más que poseerlo) de modos más sutiles, que no despertaran en el dominado un interés emancipatorio y en últimas una subversión.

Del poder del Soberano íntimamente ligado a la iglesia y como consecuencia de luchas sociales y revoluciones hemos pasado a un poder más solapado ejercido por el estado que incluso tiene como base la división de poderes de la que carecía la omnipotencia del soberano, sin embargo, este poder estatal o institucional reproduce muchos de los actos de injusticia de los sistemas previos. El poder ya no se centraliza en una grande institución, sino que se atomiza llegando a las múltiples dimensiones de la vida humana moderna, como la escuela, la familia, la fábrica, etc. Finalmente, Focault (1995), advierte sobre los mecanismos introyectados de poder y de dominación, es decir, aquellos que son implantados en el ser humano de modo que en una ilusión de libertad actúe como esperan los poderosos por voluntad propia (Foucault, 1995).

El mecanismo más viable para que aquella libertad sea tangible consiste en asumir una postura crítica reflexiva y en ocasiones emancipadora frente a la estructura o el funcionamiento de un sistema determinado, convocando a su vez, a los individuos a reconocer su potencial, para valorarlo y expresarlo, este vendría siendo el surgimiento de ese pensamiento crítico, el cual debe, además de integrar habilidades como la interpretación de experiencias o situaciones, el análisis, la evaluación, inferencia, explicación y la autorregulación, definidas a profundidad por Facione (Facione, 2007), debe contemplarse desde el panorama social y sus interacciones, con miras de que todo proceso crítico esté mediado por la participación y los intereses de aquellos individuos que por lo general conforman las minorías, pero que aun así por medio de la apropiación de ideales y su consecuente lucha conforma estructuras sólidas.

La visión crítica del mundo es un constructo, que no ha cesado en la historia de la humanidad, quizás su momento más significativo es la llamada "escuela de Frankfurt", que sienta las bases para la crítica a todo el sistema de racionalidad occidental. Esta teoría critica europea se nutre del marxismo y analiza la situación de profunda injusticia y deshumanización de la postguerra, la pregunta esencial es ¿Por qué el ser humano llegando a un nivel de racionalidad más avanzado que nunca en la historia, termina cometiendo la masacre industrial de la segunda guerra mundial? Este punto de inicio, para la reflexión sobre las consecuencias y fallas del proyecto de la modernidad va a alimentar el pensamiento de filósofos como Habermas, Sartre y el propio Foucault (1995), los cuales reconociendo la incapacidad del proyecto clásico de la ilustración para dar cuenta de los 
retos que se le imponían a la condición humana, tratan de generar teorías críticamente orientadas que propendan por la emancipación.

Habermas (1985), discípulo de lo que se ha llamado la primera escuela de Frankfurt ha propuesto una teoría de interpretación de la conducta humana y de la investigación científica que ha nutrido múltiples áreas de las ciencias sociales y en particular a la educación. La teoría de los intereses cognitivos de Habermas (1985) establece en primera medida la idea de interés, como una tendencia natural del ser humano de buscar acciones u objetos que le den placer, y en general, el placer está relacionado con las dos condiciones necesarias para subsistir y reproducirse.

Para Habermas (1985), existen 3 intereses cognitivos fundamentales que son el interés técnico, el interés practico y el interés emancipatorio. El interés técnico está asociado a la voluntad de controlar la naturaleza y está asociado a las ciencias duras regidas por el abordaje positivista y el método empírico - analítico de concebir la naturaleza, lo cual a su vez es cercano al proyecto Kantiano de la ilustración que es otro gran hito del pensamiento crítico. Sin embargo, y como buen seguidor de Adorno y Horkhaimer, Habermas (1985) reconoce las múltiples limitaciones del interés técnico para dar cuenta de los fenómenos sociales y aboga por un interés de interpretación del mundo al cual él llama interés práctico, aquí ya se ha renunciado a la pretensión de verdad absoluta, que busca la construcción colectiva del conocimiento con la clara conciencia de que este nos da más que eso, una construcción social, subjetiva y parcial del mundo.

El interés práctico desemboca en acciones que ya no solo quieren dar un resultado efectivo en términos de modificar al mundo utilitaristamente, sino que busca acciones buenas, es decir, tiene una carga moral implícita, la bondad de las acciones es relativa, pero debe estar legitimada en la interpretación colectiva de las acciones consecuencias de este interés. Finalmente, Habermas habla del interés emancipatorio, que no solo busca interpretar al mundo sino, superar los posibles sesgos de esas interpretaciones para construir activamente una realidad más justa, menos dogmática y más reflexiva. Estos tres intereses tienen su representación en teorías curriculares y modos de operar de la escuela, así, por ejemplo, el interés por controlar los tiempos y lugares de la escuela, es propio de los currículos motivados por intereses cognitivos técnicos, que resulta en el interés por indagar a los estudiantes para que construyan sus modelos personales de la realidad con ayuda de debates y e interacciones guiadas, lo que es propio de currículos motivados por intereses prácticos.

En cuanto al currículo emancipador se puede decir que busca la formación de individuos autónomos y responsables e implica una constante interacción reflexiva de estudiantes y 
docentes en cuanto las prácticas educativas para reformarlas constantemente, liberándose de los posibles sesgos de interpretación que supone el currículo práctico. Estas visiones curriculares son la base para la construcción de las pedagogías críticas latinoamericanas que se trabajarán a continuación.

\section{AMÉRICA LATINA Y LA CRÍTICA AL NEOLIBERALISMO}

La crítica al capital como elemento de dominación ha sido desde Marx (Marx, Hamburg) un elemento medular de toda teoría crítica, inclusive en América Latina no se puede prescindir de este elemento. Para Moreano es importante reconocer desde América Latina el carácter religioso del capital, la legitimación irracional de todas las prácticas que conllevan a priorizar el capital por encima de los sujetos, y su carácter mundial. El reconocimiento de las nuevas formas del capitalismo como anti vitalistas, lleva a Moreano a decir que hay una "Necesidad urgente de sustitución del socio metabolismo del capital, decimos, entonces, por otro "lazo social" que implique una refundación de la polis humana capaz de generar una lógica que vaya más allá y hacia otra cosa que a la simple morigeración (hoy ya imposible, por otra parte) de las flagrantes injusticias estructurales; así como, de la alienación y la degradación mundializadas del régimen del capital, y más inmediatamente aún, de los estructurales límites a la vida" (Moreano, 2011).

Aunque, teóricamente, el neoliberalismo vive una crisis irreversible, su presencia en la cultura no solo persiste, sino que parece afianzarse y crecer. El neoliberalismo trascendió la esfera económica y estableció una ontología propia, un modo de ser y valorar a los seres, una lógica personal e institucional que dinamita las bases mismas lo que podríamos llamar humanidad o humanismo. En el centro ya no está el ser humano, está el mercado, el capital o la empresa, y en esta cosmovisión deshumanizada no es posible construir sujetos críticos sino en contra de ese sistema. Moreano describe en detalle los pilares que legitiman al neoliberalismo en América Latina, muestra cómo se sustenta en una pseudoacademia, principalmente norteamericana que, desde instancias como FMI, emite enunciados con apariencia de académicos que determina el comportamiento económico de los países, y, por tanto, sus políticas sociales. Lo que significa que todos esos enunciados vulneran la humanidad de los más débiles de los oprimidos (Moreano, 2011). Los gobiernos aplican "principios generales" que además de ser dañinos es modo general, lo son aún más, al aplicarse sin ver las características particulares de cada país. El lugar de enunciación de estos principios hoy cada vez más desenmascarados le da al oprimido un norte para su emancipación.

El neoliberalismo devalúa los estados, se opone a la tesis del estado interventor que regula las transacciones en beneficio de sus habitantes, y por tanto, destruye uno de los 
baluartes de la humanidad, devaluar el estado es destruir el lugar político por excelencia, y reducir lo político a un apéndice de lo económico, esta naturalización del mercado destruye la idea de ser humano en sentido general, solo deja hombres individuales en la jungla del mercado (Moreano, 2011). Una jungla que se rige por principios que solo existen en la teoría, por ejemplo, el principio de la igualdad de oportunidades y el de la "mano invisible" que distribuye equitativamente los recursos en el fragor de la competencia. Joseph Stiglitz, nobel de economía ha demostrado lo falso de estos preceptos, dando una estocada al neoliberalismo en su fase teórica.

La crítica al neoliberalismo desde América Latina, debe seguir apelando al método arqueológico de Foucault, en el sentido de desentrañar los conceptos que lo constituyen en un análisis histórico de prácticas humanas, el lapso de tiempo es relativamente corto, y las herramientas teóricas abundantes. Sin embargo, ya no se trata de criticar al neoliberalismo en sí, sino a las prácticas que solapadamente persisten en la cotidianidad, hay que hacer emerger las categorías que deshumanizan la interacción humana y proponer su destrucción.

Para Dussel (1973), un elemento necesario para la emancipación latinoamericana, y en general, de los pueblos del sur epistemológico, es una crítica radical al pensamiento moderno occidental "la necesidad de un relato desde donde potenciar el pensamiento crítico, o desde donde impulsarse para superar los siguientes límites: helenocentrismo, occidentalismo, eurocentrismo, colonialismo mental y teórico; y por último, el no incluir a América Latina en la Modernidad (Russell, 1975). En este sentido, la educación se erige como un punto medular del proyecto emancipatorio, pues en su noción más básica, como mecanismo de reproducción de la cultura, puede transformar la visión del mundo de millones de estudiantes que acuden a los sistemas educativos.

\section{EDUCACIÓN PARA LA LIBERACIÓN EN LATINOAMÉRICA}

La educación para la emancipación debe ofrecer en primera medida y a lo largo de todo el proceso educativo, una "localización" que en palabras de Dussel es la acción hermenéutica por la que el observador se sitúa comprometidamente en un lugar sociohistórico, como sujeto de enunciación de un discurso, y por ello, es el lugar desde donde se hacen las preguntas problemáticas. Por esto es posible hablar de una educación crítica latinoamericana, localizando a Latinoamérica como unidad ontológica, en oposición al pensamiento moderno occidental que es esencialmente eurocéntrico. Pero para rechazar, refutar y en últimas superar ese pensamiento, es necesario conocerlo, no se puede superar el eurocentrismo sin leer a los europeos críticamente, tomando los elementos que puedan servir para el proyecto de emancipación localizado. Dussel usa el término 
marxista subsunción para referirse a lo que debe hacerse con el eurocentrismo, es decir, integrarlo para superarlo.

El discurso histórico es una herramienta política por medio de la cual los victoriosos o poderosos legitiman y reproducen su visión del mundo como algo natural. Es evidente que para tener localización crítica se requiere desmontar los sesgos que inhiben una comprensión más integral de los sucesos históricos. En el caso de América latina esto es fundamental pues, esta región no figuraba como parte de la historia antes de la modernidad y luego era concebida como algo inferior, como si aquí no hubiera ocurrido nada importante, como si los habitantes de estas tierras hubieran permanecido en un estado de animalidad desde la creación. Esta visión de la historia es la hegemónica en nuestras propias escuelas, donde se ignora el pensamiento filosófico de las comunidades indígenas y se legitima el llamado proceso civilizatorio de América.

La visión propia de las comunidades, de su historia y de su realidad, ha sido tradicionalmente subvalorada en todos los sistemas educativos que priorizan su constructo teórico estanco, esto ha silenciado la voz de los educandos como fuente de conocimiento, y en este sentido la pedagogía de la liberación de Freire y las pedagogías del sur han dado un viraje necesario y urgente para el proyecto emancipador, pues han dado voz al educando, han reconocido el conocimiento del estudiante como igual, e incluso más esencial en el proceso educativo que el conocimiento del profesor. Todo ser humano llega a la escuela con una interpretación del mundo legítima y rica en contenidos y matices "La lectura del mundo precede a la lectura de la palabra, de ahí que la posterior lectura de ésta no pueda prescindir de la continuidad de la lectura de aquél (Freire 1981)" Es esta lectura del mundo de la que no se puede prescindir en una educación para la liberación, pues de esa lectura surge el reconocimiento de las prioridades y objetivos del proceso educativo.

La reseña histórica de la evolución de las pedagogías del sur desarrollada por Mejía J. (2008), no solo da cuenta de la influencia del pensamiento político y económico en la pedagogía, sino que explica porque no se puede hablar de una sola pedagogía del sur. El proceso ontogénico de las pedagogías libertarias muestra que, aunque hay varios puntos de encuentro y criterios mínimos unificadores, no se puede concebir una pedagogía liberadora única y totalizante, esto entraría en clara contradicción con los principios que fundamentan el interés emancipatorio de la educación para la liberación, pues no puede en pro un movimiento contra hegemónico y jerárquico establecer una nueva hegemonía y jerarquía. Pero Mejía, va más allá al criticar no solo la búsqueda de un saber pedagógico totalizante y absoluto, porque reconoce al saber académico y a la propia racionalidad científica como una más de las formas de construir verdad mas no la única "Al enfrentar la pretendida universalidad de la episteme, se muestra como esta invisibilidad otras epistemes de nuestro continente" (Mejía J., 2008). 
En cuanto al currículo emancipador, se puede decir que busca la formación de individuos autónomos y responsables e implica una constante interacción reflexiva de estudiantes y docentes en cuanto las prácticas educativas para reformarlas constantemente, liberándose de los posibles sesgos de interpretación que supone el currículo práctico.

La acción derivada del interés emancipador, es en últimas praxis, si bien la palabra se ha usado en ocasiones para referirse a la acción práctica derivada del interés práctico. Se ha observado la incompatibilidad entre los currículos e incluso entre las motivaciones prácticas y técnicas, sin embargo, la praxis es compatible con el interés práctico, pero no con el técnico, pues la acción liberadora requiere como requisito una interpretación deliberada del contexto, y no se puede emancipar un ser que está restringido por ideas absolutas. Quizá el personaje más representativo de la praxis educativa sea Paulo Freire y su pedagogía de la liberación. Freire trabajó en un programa de alfabetización para adultos, práctica que, tradicionalmente, se limitaba al interés práctico, pues solo se quería alcanzar el objetivo del lecto-escritura como herramienta de trabajo.

En este contexto, Freire (2006), plantea una educación revolucionaria, que busca que los aprendices deben ser participantes activos en el programa de aprendizaje; que la experiencia de aprendizaje debe resultar significativa para el aprendiz, y que el aprendizaje debe estar orientado en sentido crítico. La experiencia solo puede resultar significativa, sí desde su comienzo hay un interés del estudiante, para eso debe existir una negociación sobre los contenidos, además, dichos contenidos deben tener una relación directa con la realidad social del estudiante. El carácter deliberativo de esta pedagogía de nuevo es un elemento del interés práctico del cual emergen problemas reales, asociados a la dominación o inequidad; por tanto, la búsqueda de la resolución de estos problemas es emancipadora.

Un currículo enfocado en la praxis debe emerger de la realimentación entre acción y reflexión, así como, de la planificación, la acción y la evaluación relacionadas recíprocamente e integradas. Debe desarrollarse en el contexto de la realidad cultural del estudiante. También debe ayudar a discernir lo natural de lo construido por la humanidad, la ciencia de la ideología, para lo cual se requiere de la discusión, que termina siendo política, en el sentido de buscar un mejor modo de vivir para todos (Freire, 2006).

\section{CONCLUSIONES}

Es evidente que la práctica educativa colombiana debería dirigirse hacia la praxis, a la educación como elemento central de la emancipación, no por esto prescindiendo de elementos técnicos del currículo, sino tomándolos como base para la interpretación 
deliberativa e incluso para la refutación. Sin embargo, el docente colombiano está fuertemente influenciado por el enfoque técnico de la educación que simplifica su trabajo mecanizándolo y librándolo de las múltiples incertidumbres que tiene la acción práctica y en particular la praxis. Si bien, se le podría perdonar a un docente su falta de conciencia y compromiso político, no puede asumir currículos técnicos sin pasarlos por un análisis práctico, ni que objetivase a sus estudiantes sin integrarlos en el proceso educativo.

Con estos elementos se conciben una educación liberadora, como una educación que emerge en relación con las condiciones del contexto, con los estudiantes como protagonistas, con un interés por debelar y desmontar los dogmas y elementos autoritarios que la componen y con una constante realimentación con la comunidad educativa y la sociedad para propender por un horizonte, que superando los constricciones del capital y del neoliberalismo, busque el buen vivir y la justicia prospectiva con la humanidad y la tierra como sujeto de derecho.

Nuestra experiencia en educación ha sido un proceso arduo, pero a la vez gratificante. Teniendo nuestra formación inicial como biólogos en Universidades de carácter público del país, (UPTC y la Universidad Nacional de Colombia) nos permitió tener una perspectiva diferente a la que se encuentra en el gremio de la educación pública, no solo en el ámbito académico disciplinar, sino que consideramos que las universidades promueven en sus estudiantes una visión crítica del mundo. Debemos decir que las perspectivas laborales en el ámbito de la ciencia pura no se compadecían de nuestros intereses humanistas y políticos, por lo que vimos en la educación la oportunidad de contribuir con nuestro conocimiento a alcanzar esa tan anhelada revolución de pensamiento.

Creemos que a muchos de los que hemos vivido esta experiencia nos ha ocurrido que nos estrellamos en nuestro idealismo inicial con una realidad muy dura en las escuelas públicas colombianas, no por la falta de recursos o la estructura del sistema educativo pues esas son condiciones que uno asume a priori y casi como el reto que nos motiva, sino por la falta de voluntad de los educandos y padres de familia, parece haber una resignación generalizada, una voluntad casi nula de educarse, de cuestionarse y por tanto de emanciparse. Inclusive, en ciertos momentos parece que el obstáculo más grande es el estudiante y no todas las otras circunstancias adversas. Parece que el docente está obligado a entretener o a buscar el mecanismo que más reduzca el esfuerzo del estudiante para que este tenga interés por la clase.

Después de una inmersión en la comunidad de trabajo, muchos hemos visto que la interacción cercana con los estudiantes, la escucha activa y la lectura de las condiciones dan pautas para un acercamiento que avive la voluntad de aprendizaje, crítico y 
emancipación, este segundo momento, nos lleva a adaptar nuestra teoría disciplinar y crítica a un lenguaje que dé cuenta de ese contexto, y en las Instituciones Educativas donde desempeñamos nuestra labor docente en los municipios de Chitaraque y Guayatá hemos encontrado un avance significativo en los estudiantes, no solo en su rendimiento académico, sino también, en su discurso personal, que demuestra cada vez más la capacidad de plantearse preguntas pertinentes y abordarlas en comunidad.

Normalmente, llegamos en la posición de profesor imponiendo unos criterios mínimos para la valoración del trabajo de los estudiantes, lo cual los constriñe, les impide plasmar un discurso personal valioso, por eso gran parte de ser docente es desaprender, dejarse sorprender por la lectura propia de cada ser humano, quitarse de la cabeza por un momento los criterios de forma que exige la academia tradicional y permitir que la humanidad de cada persona resuene en su infinita diversidad y profundidad. De esta forma, damos los primeros pasos con la responsabilidad y la posibilidad de alcanzar la emancipación desde la educación.

\section{REFERENCIAS}

Dussel, E. (1973). América Latina: dependencia y liberación. Buenos Aires, Argentina: Consejo Latinoamericano de Ciencias Sociales (CLACSO).

Facione, P. A. (2007). Pensamiento Crítico: ¿Qué es y por qué es importante?. Obtenido de: Insight Assessment - The California Academic Press: http://eduteka.icesi.edu.co/ pdfdir/PensamientoCriticoFacione.pdf

Foucault, M. (1995). Crítica y Aufklärung ¿Qué es la crítica? Revista de Filosofía-ULA, 8, 1-18.

Freire, P. (2006). Pedagogía del oprimido. Buenos Aires, Argentina: Siglo XXI Editores. Habermas, J. (1985). "Conciencia moral y acción comunicativa". Península, Barcelona: Taurus.

Marx, K. (Hamburg). Das Kapital. Hamburg: Kritik Der Politischen Oeckonomie.

Mejía J., M. R. (2008). Las pedagogías críticas en tiempos del capitalismo cognitivo Cartografiando las resistencias en educación. Medellín, Colombia: Planetapaz- Expedición Pedagógica Nacional- Programa Ondas. 
Moreano, A. (2011). Nuestra América y el pensar crítico: Neoliberalismo, cultura y sociedad. En E. Grüner (Coord.). Buenos Aires, Argentina: Clacso.

Russell, B. (1975). Principios de reconstrucción social. Traducción de E. Torralva Beci. Madrid, España: Editorial Espasa-Calpe. 